\title{
Enquête sur l'utilisation et l'effet des médicaments à base de plantes chez les patients hépatiques hospitalisés au Service de médecine et d'hépatogastroentérologie du Centre Hospitalier Universitaire (CHU) de Cocody en Côte d'Ivoire
}

\author{
KANDE Brahima1, YAO Konan ${ }^{3}$, ALLAH-KOUADIO Emile², KONE Mamidou Witabouna1,3 \\ 1 UFR Sciences de la Nature, Université Nangui Abrogoua, BP 801 Abidjan 01, Côte d'Ivoire \\ ${ }^{2}$ Service de Médecine et d'Hépato-gastroentérologie, Centre Hospitalier et Universitaire (CHU) de \\ Cocody, BP V 13 Abidjan, Côte d'Ivoire \\ ${ }^{3}$ Centre Suisse de Recherches Scientifiques en Côte d'Ivoire, 01 BP 1303 Abidjan 01, Côte d'Ivoire \\ Auteur correspondant : E-mail : konewit_sn@univ-na.ci ; mamidou.kone@csrs.ci ; Tel : + 22503488905.
}

Original submitted in on $6^{\text {th }}$ July 2018. Published online at www.m.elewa.org on $31^{\text {st }}$ October 2018 https://dx.doi.org/10.4314/jab.v130i1.9

\section{RESUME}

Objectif : Cette étude a été menée afin d'identifier les plantes et leurs produits dérivés utilisés par les patients avant leur admission au service de Médecine et d'Hépato-gastroentérologie du Centre Hospitalier et Universitaire (CHU) de Cocody en Côte d'Ivoire.

Méthodologie et résultats : Un questionnaire a été administré à ces patients au cours d'entretien semistructuré lors des enquêtes menées entre février et mai 2016. Un total de 125 patients a été enquêté dont $88 \%$ ont eu recours à la médecine traditionnelle les 12 derniers mois précédent l'enquête. Les plantes médicinales sont le plus souvent consommées par les patients pour traiter des maladies telles que le paludisme et l'asthénie avec des fréquences de citation (fc) de $45,5 \%$ et $11 \%$ respectivement. Vingt (20) plantes médicinales appartenant à 13 familles et 19 genres ont été citées. Vernonia amygdalina (Asteraceae) "feuille amère", avec une fc de 16,32 \%, Alchornea cordifolia (Euphorbiaceae) "Arbre de djeman"' et Senna occidentalis (Fabaceae) "faux kinkéliba' ( $\mathrm{fc}=12,24 \%$ ), étaient les espèces les plus utilisées.

Conclusions et application des résultats : Les patients hépatiques ont eu recours aux produits de la médecine traditionnelle. Ces résultats sont importants pour la sécurité sanitaire des consommateurs de plantes médicinales. Ils pourront contribuer à améliorer le diagnostic des maladies du foie.

Mots-clés : Centre hospitalier, Côte d'Ivoire, Hépatotoxicité, Maladies du foie, Plantes médicinales, Patients 
Kande et al., J. Appl. Biosci. 2018 Enquête sur l'utilisation et l'effet des médicaments à base de plantes chez les patients hépatiques hospitalisés du Centre Hospitalier Universitaire de Cocody en Côte d'Ivoire

Survey on the use and effect of herbal medicines on hospitalized liver patients in the Department of Medicine and Hepatogastroenterology at the University Hospital Center (UHC) of Cocody in Côte d'Ivoire

\section{ABSTRACT}

Objective: This descriptive study was conducted to identify the plants and their derivatives used by patients prior to their admission to the Medicine and Hepato-gastroenterology department of the University Hospital Center (UHC) of Cocody in Côte d'Ivoire.

Methods and Results: A semi-structured interview questionnaire was applied to these patients from February to May 2016. A total of 125 patients were interrogated, $88 \%$ of whom used traditional medicine in the last 12 months prior to the survey. The medicinal plants were mostly consumed by patients to treat diseases such as malaria and asthenia with citation frequencies (cf) of $45.5 \%$ and $11 \%$, respectively. Twenty (20) plants species belonging to 13 families and 19 genders were cited. Vernonia amygdalina (Asteraceae) "bitter leaves" with of of $16.32 \%$, Alchornea cordifolia (Euphorbiaceae) "christmas bush" and Senna occidentalis (Fabaceae) "negro coffee" (cf =12.24\%) were the most used species.

Conclusion and application of results : The liver patients have used products of traditional medicine. The results are important for the safety of consumers of medicinal plants. These results may contribute to improving the diagnosis of liver diseases.

Keywords: Hospital center, Côte d'Ivoire, Hepatotoxicity, Liver diseases, Medicinal plants, Patients

\section{INTRODUCTION}

Une frange non négligeable de la population dans les pays en développement compte encore sur les tradipraticiens, les plantes médicinales et les médicaments à base de plantes, pour leurs soins de santé de base (OMS, 1998). Ainsi, approximativement $80 \%$ de la population africaine utilisent encore la médecine traditionnelle pour ses soins de santé primaire (OMS, 2008). En outre, l'usage des ressources végétales à des fins thérapeutiques est une habitude dans toutes les cultures africaines. Des milliers d'espèces végétales font l'objet d'utilisation pour palier des problèmes de santé (Mangambu et al., 2012). Pour ces plantes médicinales, la réputation d'innocuité totale, généralement fondée sur la longue tradition d'utilisation, explique en grande partie leur utilisation (Schuppan et al., 1999). La conception populaire attribue habituellement aux plantes médicinales peu d'effets secondaires et une meilleure compatibilité avec l'organisme humain (Upadhyay et al., 2011). La sécurité et l'innocuité sont d'importants critères à prendre en compte avant l'administration des produits à base de plantes. Plusieurs rapports font état de par le monde, des effets secondaires graves enregistrés suite à l'utilisation des plantes médicinales. Au nombre de ces conséquences négatives de ces plantes figurent les dommages du foie, qui sont souvent assez graves pour rendre nécessaire une transplantation ou même encore conduire à la mort (Stournaras et al., 2015). Or, les guérisseurs, les tradipraticiens de santé et les populations n'ont pas toujours conscience de cette toxicité. Ils utilisent les plantes médicinales, dans la plupart des cas, sans inquiétude. Pourtant, certaines plantes notamment celles contenant les alcaloïdes pyrrolizidiniques (AP) et les coumarines toxiques, peuvent causer des intoxications graves. On estime qu'un grand nombre de cas d'atteinte hépatique mineure passent inaperçus. Parmi les causes, il est admis, que les drogues toxiques sont à l'origine d'environ $10 \%$ des insuffisances hépatiques aiguës et de $5 \%$ des ictères (Dossing \& Sonne 1993). Dans ce contexte, l'intérêt pour les plantes à AP et à coumarines est fortement stimulé par le fait que les alcaloïdes pyrrolizidiniques (AP) et les coumarines constituent une menace grave pour la santé humaine (Hagan et al., 1967 ; Stegelmeier et al., 1999). Ces plantes sont largement et quotidiennement utilisées par les populations pour la santé et/ou l'alimentation (Wiedenfeld, 2011 ; Roeder \& Wiedenfeld, 2013). 

chez les patients hépatiques hospitalisés du Centre Hospitalier Universitaire de Cocody en Côte d'Ivoire

Les AP et les coumarines constituent l'un des groupes les plus importants de substances végétales toxiques dans le monde. Ces $A P$ et coumarines ont une importance à cause de leur toxicité pour le foie, les reins et les poumons. Les $\mathrm{AP}$ et leurs $\mathrm{N}$-oxydes peuvent entrer dans la chaîne alimentaire comme des contaminants des céréales, via les produits animaux (lait, œuf et miel) ou sont consommés comme constituants des remèdes traditionnels à base de plantes (IPCS, 1988 ; Edgar, 2003). L'ingestion accidentelle de ces produits peut poser ainsi un risque sanitaire potentiel pour les consommateurs (Kast et al., 2014). Des épisodes de problèmes d'intoxications dues aux AP sont bien documentés pour le Pakistan (IPCS, 1988), l'Inde (Huxtable, 1989) et le

\section{MATÉRIEL ET MÉTHODES}

Cadre de l'étude et critères d'inclusion : II s'agit d'une étude transversale descriptive réalisée à partir d'un questionnaire établi, auprès des patients atteints de cancers et de cirrhoses de foie hospitalisés au service de Médecine et d'Hépato-gastroentérologie du Centre Hospitalier et Universitaire (CHU) de Cocody/Abidjan. Ce service est divisé en deux blocs: gastroentérologie et hépatologie. Une autorisation d'accès à ce service a été délivrée par la direction $\mathrm{du}$ $\mathrm{CHU}$ de Cocody. Les patients qui arrivent au $\mathrm{CHU}$ sont d'abord pris en charge par le service des urgences. Selon le premier diagnostic posé, ils sont orientés vers des services spécialisés. Ceux qui arrivent au service de Gastroentérologie et Hépatologie sont systématiquement internés dans l'un des blocs. Seuls les patients admis au bloc hépatologie ont été inclus dans la présente étude. L'inclusion a été faite au fur et à mesure de l'admission dans le service sur une période de quatre mois. Les rencontres et les échanges avec ces personnes ont eu lieu les après-midis pour ne pas perturber le travail quotidien du personnel.

Enquêtes auprès des patients: Une fiche d'enquête semi-structurée a été élaborée. Les principales informations recherchées portaient sur le statut sociodémographique, l'utilisation des plantes médicinales au cours des 12 derniers mois précédant l'enquête, les noms locaux de ces plantes, les maladies traitées avec ces espèces végétales, les raisons de consultation au service de Gastroentérologie et Hépatologie, et la consommation d'alcool ainsi que les diagnostics cliniques de maladies liées au foie. Avant de mener
Costa Rica (Van Weeren et al., 1999). II en est de même quant aux coumarines pour la Norvège (Nfsa, 2010), l'Allemagne (BfR, 2007) et de manière générale pour l'Europe (Efsa, 2008). Pour la Côte d'Ivoire, très peu de données sont disponibles sur le risque chimique des plantes dans la survenue de certaines affections du foie. L'objectif de cette étude est d'identifier les plantes et leurs produits dérivés utilisés par les patients admis au service de Médecine et d'Hépatogastroentérologie du Centre Hospitalier et Universitaire (CHU) de Cocody en Côte d'Ivoire. Ces informations recueillies auprès des patients permettront d'établir un probable lien entre les affections du foie (cirrhoses et cancers) et l'utilisation de la phytothérapie.

l'enquête, un consentement libre et éclairé du patient a été demandé après qu'il ait été informé clairement sur les motifs de l'étude. La langue utilisée pour les entretiens a été principalement le français. Dans certains cas, un interprète a servi d'intermédiaire.

Collecte et identification des espèces végétales citées: Sur la base des noms locaux, les plantes citées par les patients ont été récoltées ou achetées auprès des herboristes sur les marchés. Des herbiers ont été confectionnés et l'identification des espèces a été effectuée par les botanistes de l'UFR SN de I'Université Nangui Abrogoua. Une authentification a par la suite été effectuée par comparaison des échantillons avec ceux de l'herbier du Centre National de Floristique (CNF) de l'Université Félix Houphouët Boigny. Pour les noms des taxons, le système de nomenclature APG IV (2016) a été adopté.

\section{Traitement des données}

$>$ Pourcentage d'utilisation de la phytothérapie/pourcentage de consommation d'alcool. II se calcule selon la formule 1 :

$\%=\frac{\text { noui }}{\mathrm{N}} \times 100(1)$

Avec :

$\mathrm{n}_{\text {oui }}$ : nombre de personnes ayant utilisé les plantes médicinales ou consommant de l'alcool ;

$\mathrm{N}$ : nombre total de personnes interrogées au cours de l'enquête.

$>\quad$ Fréquences de citation des espèces végétales, des symptômes ou malaises ressentis. Elles ont été calculées selon la formule 2 : 
Kande et al., J. Appl. Biosci. 2018 Enquête sur l'utilisation et l'effet des médicaments à base de plantes chez les patients hépatiques hospitalisés du Centre Hospitalier Universitaire de Cocody en Côte d'Ivoire

$\mathbf{f c}=\frac{\mathbf{n}}{\mathrm{N}} \times 100(2)$

Avec:

fc : fréquence de citation ; $\mathrm{n}$ : nombre total d'enquêtés ;

$\mathrm{N}$ : nombre total de personnes ayant dit oui pour l'utilisation des plantes médicinales

La fréquence de citation permet ainsi d'évaluer la crédibilité des informations reçues et le niveau de connaissance des plantes de la population enquêtée (Betti, 2003). II y a crédibilité lorsque la même plante, le même genre ou la même famille est mentionné au moins deux fois pour traiter la même maladie ou affection.

\section{RESULTATS}

Caractéristiques socio-démographiques des patients enquêtés: Au total, 125 personnes ont été interrogées durant les quatre mois qu'ont duré les enquêtes. Les patients interviewés sont répartis en autant d'hommes que de femmes. L'âge moyen était de 45,5 ans (âge médian 45 ans). Le pourcentage de personnes mariées $(50 \%)$ était plus élevé que celui des célibataires $(38 \%)$. II ressort que $35 \%$ des
Analyses statistiques: Les données des enquêtes ont été saisies en utilisant le logiciel Epidata 3.1 puis transférées sur le logiciel SPSS 20.0 pour analyse. Le test de Khi-deux $\left(X^{2}\right)$ a été utilisé pour établir, s'il existe une relation entre l'usage de la phytothérapie par les patients et des paramètres tels que le sexe, la situation matrimoniale et le niveau d'instruction. Les tableaux ont été réalisés avec le logiciel SPSS 20.0 et Excel pour mieux comprendre et interpréter l'utilisation des plantes médicinales, la consommation d'alcool et les signes cliniques d'une atteinte hépatique. La signification du test est déterminée en comparant la probabilité $P<$ 0,0001 associée à la statistique du test $\mathrm{F}$ de Fisher au seuil théorique $a=0,05$ (Dagnelie, 1999).

personnes interrogées n'avaient aucun niveau d'instruction. Pour les autres (65\%), on note une proportion non négligeable de niveau primaire $(17 \%)$ et secondaire $(27 \%)$. Les patients hospitalisés étaient de confession religieuse chrétienne (61\%) et musulmane $(30 \%)$ dans la grande majorité des cas. Les caractéristiques de l'échantillon sont détaillées dans le Tableau 1.

Tableau 1: Caractéristiques socio-démographiques de l'échantillon étudié

\begin{tabular}{l|l}
\hline Caractéristiques & Proportions (\%) \\
\hline Genre & 58 \\
\hline Hommes & 42 \\
\hline Femmes & 100 \\
\hline Total & \\
\hline Age & 25 \\
\hline$\leq 35$ ans & 50 \\
\hline 36 à 55 ans & 25 \\
\hline$\geq 55$ ans & 100 \\
\hline Total & \\
\hline Situation matrimoniale & 38 \\
\hline Célibataire & 50 \\
\hline Marié & 3 \\
\hline Divorcé & 9 \\
\hline Veuf /veuve & 100 \\
\hline Total & \\
\hline Niveau instruction & 35 \\
\hline Aucun & 6 \\
\hline Coranique & 17 \\
\hline Primaire & 27 \\
\hline Secondaire & 15 \\
\hline Supérieur & 100 \\
\hline Total &
\end{tabular}


Kande et al., J. Appl. Biosci. 2018 Enquête sur l'utilisation et l'effet des médicaments à base de plantes chez les patients hépatiques hospitalisés du Centre Hospitalier Universitaire de Cocody en Côte d'Ivoire

\begin{tabular}{l|l}
\hline Religion & \\
\hline Chrétienne & 61 \\
\hline Musulmane & 30 \\
\hline Animiste & 4 \\
\hline Aucune & 5 \\
\hline Total & 100 \\
\hline
\end{tabular}

Taux d'utilisation des plantes médicinales par les patients : Sur les 125 patients inclus dans l'étude, 88 $\%$ ont eu recours à la médecine traditionnelle les 12 derniers mois précédent l'enquête. En considérant le sexe, $85 \%$ des hommes et $92 \%$ des femmes inclus dans l'étude ont utilisé des plantes médicinales. On note qu'il n'y a pas de différence significative $\left(X^{2}=\right.$ 1,$565 ; P=0,211$ ), le sexe n'influence donc pas l'utilisation de la phytothérapie. Près de $50 \%$ des personnes ayant eu recours à l'utilisation des plantes médicinales avaient un âge compris entre 36 et 55 ans. Cette tranche d'âge constituait $50 \%$ de l'échantillon de l'étude. Le pourcentage de personnes qui utilisent les plantes médicinales n'est pas significativement différent en fonction des tranches d'âge $\left(X^{2}=3,223 ; P=0,200\right)$. Cependant, le pourcentage le plus important $(97 \%)$ a été observé pour la tranche d'âge $\leq 35$ ans. Le statut matrimonial des personnes enquêtées montre que 89 $\%$ des hommes et des femmes mariés utilisent les plantes médicinales. Le pourcentage des célibataires n'est pas négligeable, soit $87,5 \%$ des personnes utilisant la phytothérapie. Ces derniers utilisent directement les produits de la phytothérapie prête à l'emploi. La presque totalité des veuf (ve) s (82\%) et des divorcés (100\%) ont recours aux plantes médicinales. La différence n'est pas significative $\left(X^{2}=\right.$ $0,984 ; P=0,805)$ quant à la situation matrimoniale. Plus de $80 \%$ des patients interrogés de chaque niveau d'instruction ont eu recours aux plantes médicinales, au cours des 12 derniers mois. Pour cet autre paramètre, la différence n'est pas significative $\left(X^{2}=3,843 ; P=\right.$ 0,428 ).

Diversité floristique des espèces végétales citées : L'enquête a permis de recenser 20 espèces végétales appartenant à 13 familles et 19 genres (Tableau 2). Les familles dominantes sont celles des Fabaceae et des Meliaceae avec trois espèces chacune. Six types biologiques correspondant majoritairement aux microphanérophytes (mp) et nanophanérophytes ( $\mathrm{np}$ ) ont été déterminés. Ils sont suivis des mésophanérophytes $(\mathrm{mP})$ et des mégaphanérophytes (MP) représentés par trois et deux espèces chacune. En ce qui concerne les types phytogéographiques, les espèces de la zone de transition savane-forêt (GC-SZ) sont les plus abondantes (10 espèces). Elles sont suivies des espèces de la Région Guinéo-Congolaise (GC, 6 espèces). Les espèces appartenant aux taxons introduits ou cultivés (i) sont les moins représentées (3 espèces).

Plantes médicinales consommées par les patients : Les plantes médicinales les plus consommées par les patients enquêtés sont Vernonia amygdalina avec une fréquence de citation (fc) de 16,32 \%, Alchornea cordifolia et Senna occidentalis avec une fc de 12,24 \% chacune, Azadirachta indica (fc $=10,20 \%$, Ocimum gratissimum et Turraea heterophylla, avec fc $=6,12 \%$ chacune. Ces plantes sont utilisées seules ou sont associées entre elles. Les plantes citées sont listées dans le Tableau 3. 
Kande et al., J. Appl. Biosci. 2018 Enquête sur l'utilisation et l'effet des médicaments à base de plantes chez les patients hépatiques hospitalisés du Centre Hospitalier Universitaire de Cocody en Côte d'Ivoire

Tableau 2 : Types biologiques et phytogéographiques des espèces végétales citées par les patients hospitalisés au service de Médecine et d'Hépato-gastroentérologie du CHU de Cocody

\begin{tabular}{|c|c|c|c|}
\hline Espèces végétales & Familles & Types biologiques & Types phytogéographiques \\
\hline Ageratum conyzoides $\mathrm{L}$. & Asteraceae & Th & GC-SZ \\
\hline $\begin{array}{l}\text { Alchornea cordifolia (Schumach. \& Thonn.) } \\
\text { Müll.Arg }\end{array}$ & Euphorbiaceae & $\mathrm{mp}$ & GC-SZ \\
\hline Alstonia boonei De Wild. & Apocynaceae & MP & GC \\
\hline Althernanthera purgens Kunth & Amaranthaceae & Th & GC-SZ \\
\hline Azadirachta indica A. Juss. & Meliaceae & $\mathrm{mP}$ & $\mathrm{i}$ \\
\hline Bambusa vulgaris Schrad. ex J.C. Wendl. & Poaceae & $\mathrm{Gr}$ & GC-SZ \\
\hline Cajanus cajan (L.) Millsp. & Fabaceae & $\mathrm{np}$ & GC-SZ \\
\hline Carica papaya L. & Caricaceae & $\mathrm{mp}$ & GC \\
\hline Khaya senegalensis (Desr.) A. Juss. & Meliaceae & $\mathrm{mP}$ & SZ \\
\hline Mangifera indica $\mathrm{L}$. & Anacardiaceae & $\mathrm{mP}$ & $\bar{i}$ \\
\hline Morinda lucida Benth. & Rubiaceae & $\mathrm{mp}$ & GC \\
\hline Moringa oleifera Lam. & Moringaceae & $\mathrm{mp}$ & GC-SZ \\
\hline Ocimum gratissimum L. & Lamiaceae & $\mathrm{np}$ & GC-SZ \\
\hline $\begin{array}{l}\text { Phyllanthus niruri var amarus (Schumach. \& } \\
\text { Thonn.) Leandri }\end{array}$ & Phyllanthaceae & $\mathrm{np}$ & GC \\
\hline Sarcocephalus latifolius (Sm.) E. A. Bruce & Rubiaceae & $\mathrm{mp}$ & GC-SZ \\
\hline Senna alata L. & Fabaceae & $\mathrm{np}$ & GC \\
\hline Senna occidentalis L. & Fabaceae & $\mathrm{np}$ & GC-SZ \\
\hline Tectona grandis L. f. & Lamiaceae & MP & $\mathrm{i}$ \\
\hline Turraea heterophylla J. Sm. & Meliaceae & $\mathrm{np}$ & GC \\
\hline Vernonia amygdalina Del. & Asteraceae & $\mathrm{mp}$ & GC-SZ \\
\hline
\end{tabular}

Tableau 3 : Fréquences de citation (\%) des plantes utilisées par les patients hospitalisés au service de Médecine et d'Hépato-gastroentérologie du CHU de Cocody

\begin{tabular}{l|l|c}
\hline Espèces végétales & Familles & Fréquence (\%) \\
\hline Ageratum conyzoides L. & Asteraceae & 2,04 \\
\hline Alchornea cordifolia (Schumach. \& Thonn.) Müll. Arg. & Euphorbiaceae & 12,24 \\
\hline Alstonia boonei De Wild. & Apocynaceae & 2,04 \\
\hline Althernanthera purgens Kunth & Amaranthaceae & 2,04 \\
\hline Azadirachta indica A. Juss. & Meliaceae & 10,20 \\
\hline Bambusa vulgaris Schrad. ex J.C. Wendl. & Poaceae & 4,08 \\
\hline Cajanus cajan (L.) Millsp. & Fabaceae & 2,04 \\
\hline Carica papaya L. & Caricaceae & 2,04 \\
\hline Khaya senegalensis (Desr.) A. Juss. & Meliaceae & 2,04 \\
\hline Mangifera indica L. & Anacardiaceae & 2,04 \\
\hline Morinda lucida Benth. & Rubiaceae & 2,04 \\
\hline Moringa oleifera Lam. & Moringaceae & 4,08 \\
\hline Ocimum gratissimum L. & Lamiaceae & 6,12 \\
\hline Phyllanthus niruri var amarus (Schumach. \& Thonn.) Leandri & Phyllanthaceae & 2,04 \\
\hline Sarcocephalus latifolius (Sm.) E. A. Bruce & Rubiaceae & 2,04 \\
\hline Senna alata L. & Fabaceae & 4,08 \\
\hline Senna occidentalis L. & Fabaceae & 12,24 \\
\hline Tectona grandis L. f. & Lamiaceae & 2,04 \\
\hline Turraea heterophylla J. Sm. & Meliaceae & 6,12 \\
\hline Vernonia amygdalina Del. & Asteraceae & 16,32 \\
\hline
\end{tabular}



chez les patients hépatiques hospitalisés du Centre Hospitalier Universitaire de Cocody en Côte d'Ivoire

Maladies traitées : Les plantes médicinales les plus consommées par les patients sont celles traitant le paludisme, avec une fréquence de citation (fc) de 45,5 $\%$ et l'asthénie ( $\mathrm{fc}=11 \%$ ). Les autres affections sont les ulcères gastriques pour $9,60 \%$ des patients, la fièvre typhoïdes ( $\mathrm{fc}=5 \%$ ), la constipation ( $\mathrm{fc}=3,75$ $\%$ ), fièvre ( $\mathrm{fc}=3 \%$ ), les hémorroïdes et crises hémorroïdaires (fc $=3 \%$ ). Un total de $23,2 \%$ des patients a consommé des plantes médicinales pour divers autres problèmes de santé comme l'anémie, les dysménorrhées, les douleurs abdominales, les ictères, les hépatites virales et aussi pour l'entretien de la grossesse.

Organes végétaux utilisés pour les préparations thérapeutiques: Diverses parties de plantes entrent dans la confection des remèdes en médecine traditionnelle. Les feuilles sont majoritairement utilisées à $41,80 \%$, ensuite viennent les écorces de tiges et les tiges à $27,70 \%$ et $17,85 \%$ respectivement. Les racines n'ont été utilisées qu'à $8,45 \%$. Pour certaines espèces végétales, la plante entière est utilisée à 4,20\%.

Techniques de préparation des remèdes et les solvants utilisés: Plusieurs techniques de préparations sont employées afin de faciliter l'administration du principe actif. II s'agit de la décoction, la macération, l'infusion, la fumigation. La décoction et la macération ont été les deux techniques les plus utilisées avec des pourcentages respectifs de $69,40 \%$ et $24,50 \%$. Quant aux solvants utilisés, l'eau et l'alcool sont majoritairement cités avec des taux de $43,75 \%$. Cependant, certains patients ont utilisé la boisson «Tonic» $(12,5 \%)$ pour leur préparation médicamenteuse.

Effets indésirables induits par la prise de plantes médicinales: La survenue des effets indésirables est notifiée par $31,2 \%$ des patients qui ont eu recours aux plantes médicinales dans les 12 derniers mois précédant l'enquête. Parmi ces patients, $48 \%$ ont eu des vomissements, $16 \%$ des nausées, $11 \%$ des jaunissements des yeux, les douleurs au ventre $(7 \%)$ et $9 \%$ des diarrhées. Chez $9 \%$ des patients, les effets indésirables étaient des symptômes tels que le manque d'appétit, les sécrétions excessives d'urines, le ballonnement du ventre, le corps chaud et les vomissements avec du sang.
Fréquences et temps de consommation des remèdes à base de plantes médicinales par les patients : La majeure partie des patients (52\%) ayant eu recours à la phytothérapie ont déclaré avoir consommé deux fois par jour les remèdes à base de plantes. Par contre, $31,20 \%$ ont consommé les remèdes trois fois par jour. Les patients ont consommé l'équivalent d'un verre de $35 \mathrm{cl}$ à chaque prise dans la grande majorité des cas $(59,32 \%)$. Parmi les patients, $27,96 \%$ ont utilisé deux verres de $35 \mathrm{cl}$ par prise tandis que $33,88 \%$ ont eu recours à un breuvage à base de plantes pour se soigner sans posologie définie. Certains malades $(25 \%)$ ont pris les phytomédicaments sur une durée d'un mois sans interruption. Au niveau des femmes, 6,45\% ont absorbé ces remèdes durant la grossesse.

Consommation d'alcool par les patients : L'enquête a relevé que plus de la moitié (56\%) des patients consomment l'alcool et $44 \%$ n'en consomment pas. Cette consommation concerne plus les hommes que les femmes $(69,86 \%$ contre $36,54 \%)$. Cette absorption de l'alcool est influencée par l'âge, le sexe, le statut matrimonial et le niveau d'instruction.

Diagnostic clinique des maladies liées au foie : Les paramètres liés aux symptômes d'une atteinte hépatique sont donnés dans le Tableau 4. Les enquêtes ont révélé que la moitié des patients $(48,8 \%)$ ont ressenti des malaises après la consommation d'un remède à base de plantes médicinales, les 12 mois précédant l'enquête. Les vomissements (43,5\%) et les salivations $(32,2 \%)$ sont les malaises prédominants par rapport à la fièvre $(6,5 \%)$, l'ictère $(8,1 \%)$, l'épigastralgie $(3 \%)$ et les autres malaises $(3,5 \%)$. Quant au temps mis par les malades avant de se rendre dans un centre de santé, il est de deux $(12,8 \%)$ à trois mois $(18,4 \%)$ de maladie. L'amaigrissement chronique est le symptôme le plus fréquent $(18,6 \%)$ pour justifier une consultation médicale et un examen approfondi par les médecins. Ce symptôme est suivi des œdèmes $(18,4 \%)$ et de l'ictère $(12,5 \%)$. Pour certains patients, la pâleur (11\%) et la distension abdominale (8,4 \%) avec le plus souvent des dilatations des veines sur l'abdomen, sont autant de signes qui ont suscité une consultation médicale. II faut noter que les symptômes d'une atteinte hépatique diagnostiqués par les médecins seraient superposables aux effets indésirables décrits par les patients. 
Kande et al., J. Appl. Biosci. 2018 Enquête sur l'utilisation et l'effet des médicaments à base de plantes chez les patients hépatiques hospitalisés du Centre Hospitalier Universitaire de Cocody en Côte d'Ivoire

Tableau 4 : Paramètres liés aux symptômes d'une atteinte hépatique

\begin{tabular}{l|l}
\hline Paramètres & Proportions (\%) \\
\hline Malaise après la consommation d'un remède & \multicolumn{2}{l}{} \\
\hline Oui & 48,8 \\
Non & 51,2 \\
\hline Types de malaises & 6,5 \\
\hline Fièvre & 43,5 \\
Vomissement & 32,2 \\
Salivation & 3,2 \\
Toux & 8,1 \\
Ictère & 3 \\
Epigastralgie & 3,5 \\
Autres & \\
\hline
\end{tabular}

Temps mis avant une consultation médicale

\begin{tabular}{l|l}
\hline Une semaine & 8 \\
Un mois & 8,8 \\
Deux mois & 12,8 \\
Trois mois & 18,4 \\
Quatre mois & 9,6 \\
Un an & 3,2 \\
Autres & 39,2 \\
\multicolumn{2}{|l}{} \\
\hline Signes cliniques pour une consultation médicale & 10 \\
\hline Sensation de blocage à l'inspiration profonde & 18,4 \\
OEdèmes & 12,5 \\
Ictère & 8,6 \\
Abcès hépatique & 18,6 \\
Amaigrissement chronique & 11 \\
Pâleur & 8,4 \\
Distension abdominale & 12,5 \\
Autres & \\
\hline
\end{tabular}

\section{DISCUSSION}

L'enquête menée au Centre Hospitalier et Universitaire (CHU) de Cocody/Abidjan, a permis de collecter des informations sur les plantes consommées et les affections survenues chez les patients hospitalisés au service de Médecine et d'Hépato-gastroentérologie. A notre connaissance, c'est la première fois qu'une telle enquête en milieu hospitalier sur l'utilisation de la phytothérapie est réalisée. La plupart des enquêtes menées en Côte d'Ivoire sur les plantes médicinales ont été conduites auprès des praticiens de la médecine traditionnelle et des populations (Diaby et al., 2011; Koulibaly et al., 2016).

Caractéristiques socio-démographiques des patients enquêtés et taux d'utilisation des plantes par les patients : La proportion des malades ayant utilisé des plantes médicinales est de $88 \%$. Cette valeur est proche des estimations de l'OMS (2008) qui donnent $80 \%$ de la population africaine utilisant encore la médecine traditionnelle pour ses soins de santé primaire (Diallo et al., 2010 ; Salhi et al., 2010 ; Dibong et al., 2011). Les patients sont issus de tous les niveaux d'instruction, allant de sans instruction au niveau d'étude supérieure. Quelques soit le niveau d'instruction, ces personnes restent attachées aux valeurs ancestrales liées à l'utilisation des plantes médicinales. Les malades ont eu recours aux phytomédicaments pour traiter des maladies fréquentes telles que le paludisme dans $85 \%$ des cas. Ce 

chez les patients hépatiques hospitalisés du Centre Hospitalier Universitaire de Cocody en Côte d'Ivoire

pourcentage élevé se comprend car cette maladie représente une pandémie dans les pays tropicaux comme la Côte d'Ivoire. Les populations ont donc d'une façon générale, recours aux plantes médicinales dans leurs pratiques quotidiennes, le plus souvent en complément des médicaments modernes. Au Burkina Faso, Giani (2007) a rapporté que $87 \%$ des personnes souffrant de paludisme se traitent à la maison avec les plantes médicinales. Au niveau de la répartition des sexes, les femmes $(92 \%)$ et les hommes $(85 \%)$ utilisent les plantes médicinales. Au Tchad, l'étude de Mogode en (2005) a plutôt montré que le sexe masculin $(91 \%)$ était dominant dans l'utilisation des plantes médicinales.

Plantes médicinales consommées par les patients: $\mathrm{Au}$ plan botanique, les familles les plus représentées sont les Asteraceae (18\%), les Euphorbiaceae (12\%) et les Fabaceae (18\%). N'guessan et al. (2009) ont montré que les familles les mieux représentées pour les soins du paludisme sont les Euphorbiaceae $(10,52 \%)$, les Asteraceae (8,77\%), les Apocynaceae (8,77 \%) et les Fabaceae $(5,26 \%)$.

Maladies traitées et organes végétaux utilisés pour les préparations thérapeutiques: Les feuilles $(41,80$ $\%)$ et les écorces du tronc $(27,70 \%)$ sont les organes les plus utilisés dans le traitement du paludisme et autres pathologies. Salhi et al. (2010) et Benkhnigue et al. (2011) sont aussi parvenus à un résultat similaire qu'ils expliquent par la facilité de récolte de ces organes. Les feuilles sont aussi le siège de la photosynthèse et parfois du stockage des métabolites secondaires responsables des propriétés biologiques. En outre, les feuilles contiennent plusieurs grands groupes de composés phytochimiques (N'guessan et al., 2009). Par ailleurs, l'avantage de l'utilisation des feuilles est que le prélèvement de $50 \%$ des feuilles n'a pas une forte incidence sur l'espèce végétale, contrairement à celui des racines et des écorces de tronc (Ouattara, 2006).

Modes de préparation des remèdes et les solvants utilisés : Pour les modes de préparation, la décoction est le mode le plus dominant $(69,40 \%)$. Dans la ville de Kénitra au Maroc, Salhi et al. (2010) ont montré que la décoction est le mode de préparation le plus utilisé $(37,6 \%)$ en médecine traditionnelle. Pour les préparations médicamenteuses, les solvants les plus utilisés sont l'eau $(43,75 \%)$ et l'alcool $(43,75 \%)$ de fabrication industrielle ou locale (Koutoukou). Ces solvants sont les plus faciles d'accès pour les populations. Ait El Cadi et al. (2012) ont rapporté l'eau comme véhicule de préparation des remèdes. Ces auteurs ont expliqué que ce recours aux extraits aqueux en médecine traditionnelle se justifie par la simplicité de l'extraction et de l'innocuité de l'eau.

Effets indésirables induits par la prise de plantes médicinales : Les principaux effets secondaires notés suite à la prise de remèdes à base de plantes sont particulièrement les vomissements (48\%), les nausées (16\%) et $16 \%$ de maux d'estomac de divers ordres (diarrhée, maux de ventre, etc.). Dans le Sud-est du Nigeria, ces mêmes effets ont été rapportés par Okafor \& Ham (1999). Selon plusieurs auteurs (Okafor \& Ham 1999 ; Posadzki, et al., 2013), les vomissements (20 $\%)$, les maux d'estomac (18\%) et les vertiges $(21 \%)$ constituent aussi les effets secondaires couramment signalés suite à l'emploi de médicaments de la médecine moderne. D'autres effets indésirables peuvent être observés et aller des réactions allergiques, de réactions cutanées type photosensibilisation, aux atteintes de différents organes (tractus gastro-intestinal, foie, reins, cœur, le système nerveux central, etc).

Fréquences et temps de consommation des remèdes à base de plantes médicinales par les patients: La fréquence de consommation des phytomédicaments est de deux fois par jour pour $52 \%$ des patients. Cette consommation a été continue pour $33,88 \%$ des enquêtés ayant recours à la phytothérapie. Selon Bénichou (1990), la fréquence d'administration d'une drogue et les intervalles entre le début et la fin d'un traitement peuvent être pris en compte pour déterminer le niveau de toxicité au sein de l'organisme.

Consommation d'alcool par les patients :L'un des facteurs importants de survenue d'une atteinte hépatique est la consommation d'alcool. Ce facteur a donc été pris en compte dans ce travail. L'enquête a révélé parmi les patients hospitalisés au service visité au $\mathrm{CHU}$ de Cocody, 44 \% ne consomment pas l'alcool. Ces patients non consommateurs d'alcool présentent un intérêt dans ce travail afin de pouvoir établir un lien entre les plantes et les atteintes hépatiques. Selon Biour et al. (1998), les hépatites d'origine médicamenteuse couvrent la quasi-totalité des pathologies hépatiques et concernent, à l'heure actuelle, plus de 1100 médicaments dans le monde. La survenue d'hépatites due à l'utilisation des plantes médicinales ne doit donc pas être négligée.

Diagnostic clinique des maladies liées au foie: Concernant les signes cliniques pour une consultation médicale, l'amaigrissement chronique est le symptôme le plus fréquent $(18,6 \%)$ mentionné par les patients. II 
est suivi des œdèmes (18,4 \%) et de l'ictère $(12,5 \%)$. Selon les patients, la pâleur $(11 \%)$ et la distension abdominale $(8,4 \%)$ sont plusieurs signes qui ont justifié une consultation médicale. Une fois à l'hôpital, l'interrogatoire conduit par le personnel de santé s'est attaché à préciser la date des premières

\section{CONCLUSION}

Les résultats de l'enquête menée auprès des patients atteints de cancers et de cirrhoses de foie hospitalisés au service de Médecine et d'Hépato-Gastroentérologie du Centre Hospitalier et Universitaire (CHU) de Cocody montrent qu'il pourrait bien exister un lien entre la

\section{REMERCIEMENTS}

Les auteurs sont reconnaissants à la direction du Centre Hospitalier et Universitaire ( $\mathrm{CHU}$ ) de Cocody pour l'accès au service de Médecine et d'Hépato-

\section{REFERENCES BIBLIOGRAPHIQUES}

Ait El Cadi M., Makram S., Ansar, M. Khabbal Y., Alaoui K., Faouzi M.A, Y. Cherrah Y. \& Taoufik J. (2012). Activité anti-inflammatoire des extraits aqueux et éthanolique de Zygophyllum gaetulum. Annales Pharmaceutiques Françaises 70 (2), pp 113-116.

Andreu V., Mas A., Bruguera M., Salmeron J.M., Moreno V., Nogue S. \& Rodes J. (1998). A common cause of severe acute hepatotoxicity. Journal of Hepatology 29, pp 394-397.

APG (Angiosperm phylogeny Group) (2016). The Linnean Society of London, Botanical Journal of the Linnean Society, 181, pp 1-20.

Bénichou C. (1990). Criteria of drug-induced liver disorders. Report of an international consensus meeting. Journal of Hepatology 11, pp 272-276.

Benkhnigue O., Zidane L., Mohamed F., Houda E., Atmane R. \& Allal D. (2011). Etude ethnobotanique des plantes médicinales. Acta Botanica Barcinonensia 53, pp 191-216.

Betti J.L. (2003). Plantes utilisées pour soigner le paludisme dans la réserve de Dja au Cameroun. Revue de Médecines et Pharmacopées Africaines 17, pp 121-130.

BfR (2007). Consumers may take in larger amounts of coumarin from cosmetics, too. BfR Press Release 24/2007, 20 p.

Biour M., Poupon R., Grangé J.D., Chazouillères O. \& Jaillon P. (1998). Hépatotoxicité des médicaments; $11^{\mathrm{e}}$ mise à jour du fichier manifestations, qu'elles soient biologiques ou cliniques. Les symptômes non spécifiques, tels qu'une asthénie, des nausées, des douleurs abdominales ou une fièvre sont aussi pris en compte (Larrey 1997 ; Andreu et al., 1998).

survenue de maladies du foie et la consommation des produits de la médecine traditionnelle d'origine végétale. La recherche de composés hépatotoxiques tels que les alcaloïdes pyrrolizidiniques et les coumarines est en cours.

Gastroentérologie du Centre Hospitalier et Universitaire $(\mathrm{CHU})$ de Cocody.

bibliographique des atteintes hépatiques et des médicaments responsables. Gastroenterology Clinical Biology 22, pp 10041044.

Dagnelie P. (1999). Théories et méthodes statistiques, presse agronomique de Gembloux (2e éd.). Gembloux (Belgique) 378 p.

Diallo D., Guissou I.P., Haïdara M., Tall C. \& Kasilo O.M.J. (2010). Recherche sur la médecine traditionnelle africaine : hypertension, Observatoire Africain de la santé.

Dibong S.D., Mpondo-Mpondo E., Ngoye A. \& Kwin N.F. (2011). Plantes médicinales utilisées par les populations bassa de la région de Douala au Cameroun. International Journal of Biological and Chemical Sciences 5, pp 11051117.

Diaby B., Kroa E., Niaré A., Coulibaly D., Traoré Y., Giani S., Coulibaly A., Niaré B., Guindo A., Keita M.T., Sylla A.O., Sanogo R. \& Doumbo O.K. (2011). Connaissances, attitudes et pratiques des tradipraticiens de la sante de la ville d'Abidjan sur le paludisme. Mali medical 26, (3), pp 8-12.

Dossing M. \& Sonne J. (1993). Drug-induced hepatic disorders. Incidence, management, and avoidance. Drug Safety 9, pp 441-449.

Edgar J.A. (2003). Pyrrolizidine alkaloids and food safety. Chemical-Australian 70, pp 4-7. 
Kande et al., J. Appl. Biosci. 2018 Enquête sur l'utilisation et l'effet des médicaments à base de plantes chez les patients hépatiques hospitalisés du Centre Hospitalier Universitaire de Cocody en Côte d'Ivoire

EFSA (2008). Coumarin in flavourings and other food ingredients with flavouring properties. EFSA Journal 793, pp 1-15.

Giani S. (2007). Paludisme et Médecine traditionnelle. Aidemet Ong, Bamako, $2 \mathrm{p}$.

Hagan E.C., Hansen W.H., Fitzhugh O.G., Jenner P.M., Jones W.I., Taylor, J.M \& Brouwer J.B. (1967). Food flavourings and compounds of related structure. II. Subacute and chronic toxicity. Food and Cosmetics Toxicology 5, pp 141157.

Huxtable R. (1989). Human health implications of pyrrolizidine alkaloids and herbs Containing them. In: Cheeke, P. R., ed. Toxicants of Plant Origin, Volume 1. Boca Raton: CRC Press Inc., pp 41-86.

IPCS (1988). Pyrrolizidine alkaloids. Environnemental Health Criteria, 80. World Health Organization, Geneva.

Kast C., Dübecke A., Kilchenmann V., Bieri K., Böhlen M., Zoller O., Beckh G. \& Lüllmann C. (2014). Analysis of Swiss honeys for pyrrolizidine alkaloids. Journal of Apicultural Research 53 (1): DOI 10.3896/IBRA pp.1.53.1.

Koulibaly A., Monian M., Ackah J.A.A.B., Kone M.W. \& Traore K. (2016). Étude ethnobotanique des plantes médicinales : cas des affections les plus fréquentes d'une région agricole Daloa (Centre Ouest, Côte d'Ivoire). Journal of Animal \& Plant Sciences 31 (2), pp 50215032.

Larrey D. (1997). Hepatotoxicity of herbal remedies. Journal of Hepatology 26, pp 47-59.

Mangambu M., Van Diggelen R., Mwanga Mwanga JC., Ntahobavuka H. Malaisse F. \& Robbrecht E. (2012). Etude ethnoptéridologique, évaluation des risques d'extinction et stratégies de conservation aux alentours du Parc National de Kahuzi Biega en R.D. Congo. International Journal of Tropical Ecology and Geography 36 (1/2), pp 137-145.

Mogode D.J. (2005). Étude phytochimique de Cassia nigrans Vahl (Caesalpiniaceae) utilise dans le traitement des dermatoses au Tchad. Thèse Doctorat Université Bamako, 234 p. www.keneya.net/fmpos/theses/2005/pharma/0 5P31/pdf/05P31.pdf

N'Guessan K., Tra Bi F.H. \& Koné M.W. (2009). Étude ethnopharmacologique de plantes antipaludiques utilisées en médecine traditionnelle chez les Abbey et Krobou
d'Agboville (Côte d'lvoire).

Ethnopharmacologia 44, pp 42-50.

NFSA (2010). Risk assessment of coumarin intake in the Norwegian population: Opinion of the Panel on food additives, flavourings, processing aids, materials in contact with food and cosmetics of the Norwegian scientific committee for food safety. Nfsa Press release 09/405-2 final.

Okafor J.C. \& Ham R. (1999). Identification, utilisation et conservation des plantes médicinales dans le sud-est du Nigeria. Thèmes de la biodiversité africaine $3,8 \mathrm{p}$.

OMS (1988). Rapport Biennal du Directeur Général. Genève: National capabilities and needs In aspects of environmental health in rural and urban development and housing. Organisation mondiale de la Santé, Genève, (document WHO/EHE/AUD/88.1).

OMS (2008). Traditional medicine. Fact sheet $\mathrm{N}^{\circ} 134$. Consulté le 2/05/2014 de http://www.who.int/mediacentre/factsheets/fs1 34/en/index.html

Ouattara D. (2006). Contribution à l'inventaire des plantes médicinales significatives utilisées dans la région de Divo (sud forestier de la Côte-d'lvoire) et à la diagnose du poivrier de Guinée : Xylopia aethiopica (Dunal) A. Rich. (Annonaceae). Thèse de Doctorat de I'Université de Cocody-Abidjan (Côte-d'Ivoire), UFR Biosciences, Laboratoire de Botanique, $184 \mathrm{p}$.

Posadzki P., Watson L.K. \& Ernst E. (2013). Adverse effects of herbal medicines: an overview of systematic reviews. Clinical medicine 13 (1), pp 7-12.

Roeder E. \& Wiedenfeld H. (2013). Plants containing pyrrolizidine alkaloids used in the Traditional Indian Medicine-including Ayurveda. Die Pharmazie 68 (2), pp 83-92.

Salhi S., Fadli M., Zidane L. \& Douira A. (2010). Études floristique et ethnobotanique des plantes médicinales de la ville de Kénitra (Maroc). Lazaroa 31, pp 133-146.

Schuppan D., Jia J.D., Brinkhaus B. \& Hahn E.G. (1999). Herbal products for liver diseases: a therapeutic challenge for the new millennium. Hepatology 30, pp 1099-1104.

Stegelmeier B.L., Edgar J.A., Colegate S.M., Gardner D.R., Schoch T.K., Coulombe R.A. \& Molyneux R.J. (1999). Pyrrolizidine alkaloid 
Kande et al., J. Appl. Biosci. 2018 Enquête sur l'utilisation et l'effet des médicaments à base de plantes chez les patients hépatiques hospitalisés du Centre Hospitalier Universitaire de Cocody en Côte d'Ivoire

plants, metabolism and toxicity. Journal of Natural Toxins 8, pp 95-116.

Stournaras E. \& Tziomalos K. (2015). Herbal medicinerelated hepatotoxicity. World Journal of Hepatology 7 (19), pp 2189-2193.

Upadhyay H.C., Saini D.C. \& Srivastava S.K. (2011). Phytochemical Analysis of Ammannia multiflora. Research Journal of Phytochemistry 5, pp 170-176.

Van Weeren P.R., Morales J.A., Rodríguez L.L., Cedeño H., Villalobos J., \& Poveda L.J.
(1999). Mortality supposedly due to intoxication by pyrrolizidine alkaloids from Heliotropium indicum in a horse population in Costa Rica: a case report. Veterinary Quarterly 21, pp 59-62.

Wiedenfeld H. (2011). Plants containing pyrrolizidine alkaloids: toxicity and problems. Food Additives \& Contaminants Part A 28, pp 282292. 